\title{
Predictors of Satisfaction with Obstetric Care in High-risk Pregnancy: The Importance of Patient-Provider Relationship
}

\author{
Sheera F. Lerman - Golan Shahar · Kathryn A. Czarkowski • \\ Naamit Kurshan · Urania Magriples · Linda C. Mayes · C. Neill Epperson
}

Published online: 30 October 2007

(C) Springer Science+Business Media, LLC 2007

\begin{abstract}
The study set out to examine the predictive effects of patients' emotional distress and their relationships with their health care providers on satisfaction with obstetric services in high-risk pregnancies. Participants were 104 pregnant women with a history of recurrent losses, fetal demise, previous or current fetal genetic abnormality, advanced maternal age, or obstetric or medical complications of the present pregnancy. Self-report measures of emotional distress and the quality of their relationships with their medical provider were administered. Hierarchical multiple regression analyses were conducted to assess the predictive effect of these variables on satisfaction with services. Provision of information, constructive communication, and good relationships predicted elevated satisfaction with health services. Provision of information also buffered against the adverse effect of emotional distress on satisfaction with health services. These findings elucidate the central role of providerpatient interaction, particularly as it is related to provision of information, in high-risk pregnancy.
\end{abstract}

S. F. Lerman $(\bowtie) \cdot$ G. Shahar

Department of Psychology, Ben-Gurion University of the Negev, Beer-Sheva 84105, Israel

e-mail: sheera@bgu.ac.il

G. Shahar · K. A. Czarkowski - N. Kurshan - C. N. Epperson Department of Psychiatry, Yale University School of Medicine, New Haven, CT, USA

U. Magriples · C. N. Epperson

Department of Obstetrics, Gynecology and Reproductive Sciences, Yale University School of Medicine, New Haven, CT, USA

L. C. Mayes

Yale Child Study Center, New Haven, CT, USA
Keywords High-risk pregnancy · Patient-doctor relationships - Emotional distress . Medical information · Women's health

Pregnant women at high-risk for adverse pregnancy outcome secondary to previous or current pregnancy complications are likely to experience considerable emotional distress (Kurki, Hiilesmaa, Raitasalo, Mattila, \& Ylikorkala, 2000; Orr \& Miller, 1995). Phillips, Dennerstein, and Farish (1996) reported that approximately onethird of inpatient obstetric-gynecology patients scored at or above the 75th percentile on the Spielberger State-Trait Anxiety Inventory (STAI). This elevated level of emotional distress, often coupled with a practitioners' failure to detect their patients' distress, gives rise to patient dissatisfaction with health services (Appleby, Fox, Shaw, \& Kumar, 1989; Clarke \& Smith, 1995; Dunsis \& Smith, 1996; Hrasky \& Morice, 1986; Phillips \& Dennerstein, 1993; Phillips et al., 1996; Stewart \& Lippert, 1988).

A host of factors are known to contribute to satisfaction with obstetric care, including type of communication received, physician gender (Roter, Geller, Bernhardt, Larson, \& Doksum, 1999), and patients' emotional state. Provision of pertinent information is associated with elevated satisfaction with service (Harrison, Kushner, Benzies, Rempel, \& Kimak, 2003), as is the utilization of a warm, reassuring, often non-formal, treatment approach (Di Blasi, Harkness, Ernst, Georgiou, \& Kleijnen, 2001).

Building on these findings, the current investigation sought to examine the unique effect of patients' emotional distress on relationships with obstetric providers in a highrisk obstetric service. It was anticipated that women seen in this specialty service would be likely to have additional 
concerns about pregnancy outcome secondary to a previous adverse outcome or problem with the present pregnancy. Three aspects of patient-provider relationships were examined: the quality of medical information provided, the quality of communication, and the quality of the relationships. The hypotheses were as follows:

H1 Distress would predict lower satisfaction with obstetric care.

H2 Elevated levels of the quality of information, communication, and relationships would be associated with greater satisfaction with obstetric care.

H3 Each of the three patient-provider relationship aspects would interact with patient distress, such that the adverse effect of distress would be particularly pronounced under low, but not high, levels of the relationship variables.

\section{Methods}

This research was conducted at Yale Maternal-Fetal Medicine (MFM) outpatient sites and approved by the Yale University School of Medicine Human Investigation Committee. The Yale MFM program provides high-risk maternity services to women with a history of pregnancy risks and complications or current medical complications during pregnancy. In addition to these services, the program also offers optional prenatal classes in the second trimester, which are not covered by insurance. Participants in this study were women presenting with a history of recurrent losses, previous second or third trimester pregnancy loss, fetal demise, previous or present fetal genetic abnormality, advanced maternal age and/or other obstetric or medical complications of pregnancy. One hundred and four patients were enrolled out of approximately 200 women approached during the two-year recruitment period. Demographic variables such as mother's age, gestation age, number of past pregnancies and number of living children are presented in Table 1 . Seventy percent of the women in the sample were Caucasian, 4\% Asian, 3\% African American, $2 \%$ Hispanic and $21 \%$ were of mixed or unknown race.

Women were approached while waiting for their clinician and asked whether they would be interested in

Table 1 Means, standard deviations and range of demographic variables $(N=104)$

\begin{tabular}{lcll}
\hline Variables & Mean & $S D$ & Range \\
\hline Mother's age & 33.67 & 5.12 & $19-45$ \\
Gestation age (weeks) & 14.74 & 3.42 & $8-22$ \\
Past pregnancies & 2.6 & 1.43 & $0-7$ \\
Living children & 1.15 & 1.1 & $0-6$ \\
\hline
\end{tabular}

participating in a study focusing on their feelings about their patient-practitioner relationship. Participants who agreed to the study provided written informed consent. They were then given a series of questionnaires and reimbursed \$25 for their time. Information on women who declined participation in the study was unable to be obtained due to the Health Insurance Portability and Accountability Act (HIPAA), which went into effect in 2003. Thus without a signed consent form these women's medical records were not available for review.

\section{Measures}

The relationship between patient and practitioner was measured by multiple scales.

The first scale was the Patient Reactions Assessment (PRA; Galassi, Schanberg, \& Ware, 1992), which is a 15item self-report measure that assesses three dimensions of patient-practitioner relationships: information, communication and relationships. Participants were asked to rate on a 7-point Likert scale to what extent they agreed or disagreed with the listed statements $(1=$ very strongly disagree, $7=$ very strongly agree). Sample items for the dimensions were as follows: information (e.g., "Providers in this clinic explained my medical plan") Cronbach's $\alpha=$ .89; quality of communication (e.g., "It is hard for me to ask how my treatment is going") Cronbach's $\alpha=.83$; and affective quality of the relationship (e.g., "Providers in this clinic really respect me") Cronbach's $\alpha=.86$.

Satisfaction with health service was assessed via the eight-item Client Satisfaction Questionnaire (CSQ-8; Attkisson \& Greenfield, 1996; Attkisson \& Greenfield, 1999), which is perhaps the most commonly used instrument for the assessment of patients' satisfaction with health care services (Maruish, 2002). Extensive research supports the reliability, validity, and utility of this instrument (Attkisson \& Greenfield, 1996; Attkisson \& Greenfield, 1999; Maruish, 2002) in the current study Cronbach's $\alpha=.93$.

Patients' distress was assessed using the Perceived Stress Scale (PSS), 14-items (Cohen, Kamarck, \& Mermelstein, 1983). This measure was designed to assess the degree to which situations in one's life are perceived as stressful. Patients were asked to rate the frequency in which they had certain thoughts and feelings in the last month on a 5-point Likert scale (ranging from $0=$ "Never" to $4=$ "Very often"). This is an empirically established index (Cohen, Kessler, \& Gordon, 1995 ) in the current study Cronbach's $\alpha=.80$.

\section{Statistical Approach}

A hierarchical multiple regression (HMR) analysis was conducted to assess the predictive effect of distress and 
patient-provider relationships. The first MR Block included four predictors of satisfaction with services: distress, and the three PRA subscales. Block 2 included interactions between distress and the three PRA subscales. Given that there were clear hypotheses regarding the direction of effects, one-tailed tests of statistical significance $(p<.05)$ were employed. The statistical software package STATISTICA 7.0 (StatSoft Inc., Tulsa, OK, 2004) was used for all data analysis.

\section{Results}

Means, standard deviations and intercorrelations between the study variables are presented in Table 2. Notably, the means of satisfaction with services and patient-provider relationships were very high in this sample. This provides the overall context within which our findings should be interpreted. Namely, we were investigating a largely satisfied group of high-risk obstetrical patients.

As seen in Table 2, satisfaction with services correlated significantly with all the subscales of the PRA: information, communication and relationships $(r=.49, p<.0001$; $r=.40, p<.001 ; r=.41, p<.001$, respectively) and with age $(r=.21, p<.05)$. Age also correlated negatively with distress $(r=-.23, p<.05)$ and positively with the communication subscale of the PRA $(r=.27, p<.01)$.

In Table 3 we present the results of the HMR. The predictors in Block 1 explained $34 \%$ of the variance of satisfaction with care $\left(R^{2}=.34, F_{[7,96]}=8.53, p<.001\right)$. Statistically significant predictors in this block were the three subscales of the PRA: information $(\beta=.35$, $p<.001)$, communication $(\beta=.18, p=.05)$, and relationships $(\beta=.26, p<.01)$. Patients who reported that providers supplied adequate information and facilitated constructive communication and relationships also reported more satisfaction with care.
Block 2, which included interactions between the three PRA scales and distress, added 5\% to the explained variance of satisfaction with services $\left(R^{2}=.39, \quad F_{[10}\right.$, $\left.{ }_{93]}=7.61, p<.001\right)$. The only statistically significant interaction was between distress and PRA-information ( $\beta=.16, p=.05$ ). Probing this interaction following the recommendation of Aiken and West (1991), an interesting pattern was found, which is depicted in Fig. 1. As shown in this figure, patients' distress was negatively associated with satisfaction with health care under low (1 SD below the mean) but not high (1 $S D$ above the mean) levels of information. Thus, it may be said that provision of information buffers against the adverse effect of emotional distress on satisfaction with health care. As well, from the point of view of the association between provision of information and satisfaction with health care, the pattern presented in Fig. 1 suggests that this association is positive and strong under high, but not low, distress (1 SD above

Table 3 Results of the hierarchical multiple regression (HMR) analysis predicting satisfaction with care

\begin{tabular}{lcr}
\hline Variable & $B$ & $T$ \\
\hline Block 1 $\left(R^{2}=.34\right)$ & -.09 & -1.08 \\
Distress & $.35^{* * *}$ & 4.02 \\
PRA information & $.18^{*}$ & 1.94 \\
PRA communication & $.26^{* *}$ & 2.96 \\
PRA relationships & .09 & 1.03 \\
Age & -.02 & -.21 \\
Race & .05 & .60 \\
Gestation age & & \\
Block 2 $\left(R^{2}=.39\right)$ & $.16^{*}$ & 1.66 \\
Distress $\times$ PRA information & .12 & 1.20 \\
Distress $\times$ PRA communication & .08 & .92 \\
Distress $\times$ PRA relationships &
\end{tabular}

Note: $* p<.05, * * p<.01, * * * p<.001$ (one tailed tests)

Table 2 Intercorrelations, means, standard deviations and range of the study variables $(N=104)$

\begin{tabular}{llllll}
\hline Variables & Satisfaction & Distress & PRA information & PRA communication & PRA relationships \\
\hline Satisfaction & 1.00 & & & & \\
Distress & -.13 & 1.00 & & & \\
PRA information & $.49^{* * *}$ & -.09 & 1.00 & 1.00 & 1.00 \\
PRA communication & $.40^{* * *}$ & .09 & $.29^{* *}$ & $.36^{* * *}$ & -.02 \\
PRA relationships & $.41^{* * *}$ & .01 & $.26^{* *}$ & $.27^{* *}$ & 6.00 \\
Age & $.21^{*}$ & $-.23^{*}$ & .16 & 6.28 & 1.32 \\
Mean & 28.7 & 25.12 & 5.9 & 1.00 & 33.67 \\
$S D$ & 3.9 & 7.40 & 1.23 & $2-7$ & 5.12 \\
Range & $13-32$ & $8.61-44$ & $1.8-7$ & & $19-45$ \\
\hline
\end{tabular}

$* p<.05, * * p<.01, * * * p<.001$ 


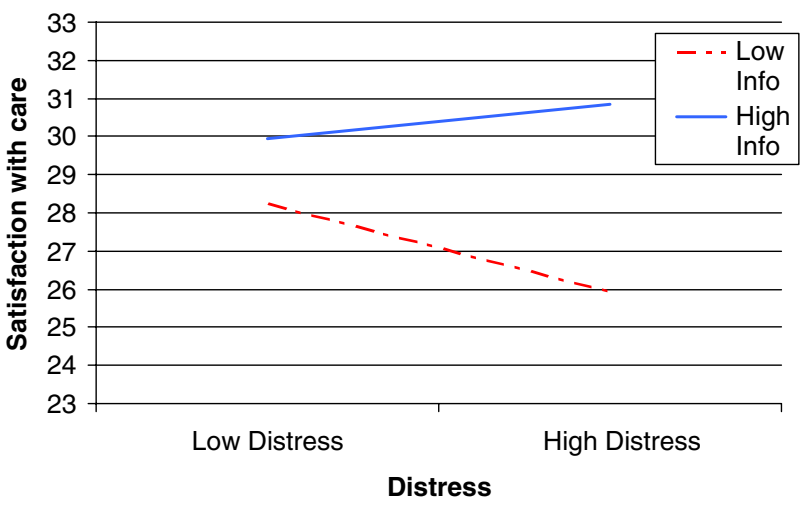

Fig. 1 Satisfaction with services as a function of distress and information

and below the mean, respectively). Patients' age, race (coded as a dummy variable differentiating between Caucasians ["1"] and non-caucasians ["0"]), and gestation age were statistically controlled for in both blocks.

\section{Discussion}

While it was hypothesized (H1) that distress alone would predict lower levels of satisfaction with obstetric care, H1 was not supported by the data. In contrast, consistent with hypothesis (H2), it was found that provision of information, constructive communication, and good relationships, all predicted elevated satisfaction with health services. These findings extend previous research demonstrating the importance of providing patients with information regarding their care in a warm and reassuring fashion (Di Blasi et al., 2001; Harrison et al., 2003).

Moreover, in partial support of our third hypothesis (H3), patient distress interacted with one of the three patient-provider relationships, namely, the information aspect. The pattern of this interaction was such that provision of information buffered, or protected, against the adverse effect of distress on satisfaction with care. Of note, however, provision of information was only associated with satisfaction with health care when distress is high, but not when it is low. This synergetic action of patients' distress with perception of information received from their provider is important, since high-risk pregnant women are likely to be considerably anxious (Kurki et al., 2000; Orr \& Miller, 1995), and OBGYN practitioners could carefully attend to, and monitor, this anxiety as they deliver medical information.

The most salient limitation of the current study is likely related to the fact that, as a whole, these study participants were very satisfied with their medical care. Subsequently, the present design does not address whether or not the overall level of high patient satisfaction also influenced how patients rated their physicians' behaviors though it is reasonable to expect such a bias. If so, it would be expected that these relationships would be even stronger if data had been obtained regarding ideal or expected physician behavior prior to women having experience with a specific clinical setting. It might also be true that evaluating a greater range in satisfaction/dissatisfaction by extending these methods to other women's health settings such as fertility or oncology clinics might yield different results. In addition, it can not be ruled out that women who were pleased with their medical care in this setting were more likely to participate in this study.

Nonetheless, even with these caveats, the findings of this study suggest that patients are satisfied with their care not solely based on an optimal outcome. Other critical factors are the interpersonal characteristics of their caregivers and how much their caregivers are willing to engage them as informed collaborators in their own care. Further, these findings indicate that emotionally distressed patients are not necessarily dissatisfied with their care but rather that practitioners may diminish their patients' anxiety with appropriate and well-timed information regarding their medical care and condition. It is important to note that these findings are suggestive but not conclusive and further research is needed in order to establish causational relationships between these variables. Finally, the finding that provision of information is particularly effective under high, but not low, distress suggests that attending to a patient's mental state and needs is essential not only for comprehensive care but also to insure the effectiveness of delivering pertinent information. Patients should be screened for distress when they come to receive health care services and providers of care must be trained in order to use this information to improve relationships with their patients and provide more accurate and comprehensive information. In addition, clinical psychologists should initiate, implement and oversee consultation-liaison services especially in clinics where patients are more prone to high levels of distress.

Taken together, these findings reinforce previous information on the importance of psychosocial training for OBGYN practitioners working with women with high-risk pregnancies.

Acknowledgments This study was funded by a grant from the American Psychoanalytic Association to Golan Shahar, Ph.D. (Principal Investigator), C. Neill Epperson, M.D., and Linda Mayes, M.D. (Co-Principal Investigators). The authors wish to thank Christine Dunn, B.S., and Sara Beyor, B.S., for their diligence and organization while collecting and managing the data for this project. 


\section{References}

Aiken, L. S., \& West, S. G. (1991). Multiple regression: Testing and interpreting interactions. Newbury Park, CA: Sage.

Appleby, L., Fox, H., Shaw, M., \& Kumar, R. (1989). The psychiatrist in the obstetric unit Establishing a liaison service. British Journal of Psychiatry, 154, 510-515.

Attkisson, C. C., \& Greenfield, T. K. (1996). The Client Satisfaction Questionnaire (CSQ) Scales and the Service Satisfaction Scale30 (SS-30). In L. I. Sederer \& B. Dockey (Eds.), Outcomes assessment in clinical practice (pp. 120-127). Baltimore, MD: Williams \& Witkins.

Attkisson, C. C., \& Greenfield, T. K. (1999). The UCSF Client Satisfaction Scales: I. The Client Satisfaction Questionnaire-8. In M. E. Maruish (Eds.), The use of psychological testing for treatment planning and outcomes assessment (pp. 1222-1346). Mahwah, NJ: Lawrence Erlbaum Associates.

Clarke, D. M., \& Smith, G. C. (1995). Consultation-liaison psychiatry in general medical units. Australian and New Zealand Journal of Psychiatry, 29, 424-432.

Cohen, S., Kamarck, T., \& Mermelstein, R. (1983). A global measure of perceived stress. Journal of Health and Social Behavior, 24, 385-396.

Cohen, S., Kessler, R., \& Gordon, L. (1995). Measuring stress. Oxford: Oxford University Press, Inc.

Di Blasi, Z., Harkness, E., Ernst, E., Georgiou, A., \& Kleijnen, J. (2001). Influence of context effects on health outcomes: A systematic review. Lancet, 357, 757-762.

Dunsis, A., \& Smith, G. C. (1996). Consultation-liaison psychiatry in an obstetric service. Australian and New Zealand Journal of Psychiatry, 30, 63-73.

Galassi, J. P., Schanberg, R., \& Ware, W. B. (1992). The Patient Reactions Assessment: A brief measure of the quality of the patient-provider medical relationship. Psychological Assessment, 4, 346-351.
Harrison, M. J., Kushner, K. E., Benzies, K., Rempel, G., \& Kimak, C. (2003). Women's satisfaction with their involvement in health care decisions during a high-risk pregnancy. Birth, 30, 109-115.

Hrasky, M., \& Morice, R. (1986). The identification of psychiatric disturbance in an obstetric and gynaecological population. Australian and New Zealand Journal of Psychiatry, 20, 63-69.

Kurki, T., Hiilesmaa, V., Raitasalo, R., Mattila, H., \& Ylikorkala, O. (2000). Depression and anxiety in early pregnancy and risk for preeclampsia. Obstetrics and Gynecology, 95, 487-490.

Maruish, M. E. (2002). Psychological testing in the age of managed behavioral health care. Mahwah, NJ: Lawrence Earlbaum Associates.

Orr, S. T., \& Miller, C. A. (1995). Maternal depressive symptoms and the risk of poor pregnancy outcome. Review of the literature and preliminary findings. Epidemiologic Reviews, 17, 165-171.

Phillips, N., \& Dennerstein, L. (1993). The psychiatrist in an obstetric/gynaecology hospital: Establishing a consultation-liaison service. Australian and New Zealand Journal of Psychiatry, 27, 464-471.

Phillips, N., Dennerstein, L., \& Farish, S. (1996). Psychological morbidity in obstetric-gynecology patients: Testing the need for expanded psychiatry services in obstetric-gynecology facilities. Australian and New Zealand Journal of Psychiatry, 30, 74-81.

Roter, D. L., Geller, G., Bernhardt, B. A., Larson, S. M., \& Doksum, T. (1999). Effects of obstetrician gender on communication and patient satisfaction. Obstetrics and Gynecology, 93(5 Pt 1), 635641.

StatSoft, Inc. (2004). STATISTICA (data analysis software system). Tulsa: http://www.startsoft.com

Stewart, D. E., \& Lippert, G. P. (1988). Psychiatric consultationliaison services to an obstetric and gynecology department. Canadian Journal of Psychiatry, 33, 285-289. 